\title{
APPLICABLE SOLUTION FOR A CLASS OF ORDINARY DIFFERENTIAL EQUATIONS WITH SINGULARITY
}

\author{
N. AMEER AHAMAD* \\ Department of Mathematics, Faculty of Science, University of Tabuk, \\ P.O. Box 741, Tabuk 71491, Saudi Arabia \\ *Corresponding author: n.ameer1234@gmail.com
}

\begin{abstract}
Boundary value problems arise in many real applications such as nanofluids and other areas of applied sciences. The temperature/nanoparticles concentration are usually expressed as singular $2^{\text {nd }}$ order ODEs. So, it is a challenge to obtain the exact solution of these problems due to the difficulty of the singularity encountered in the governing equations. By means of a suitable transformation, a direct approach is introduced to solve a general class of $2^{\text {nd }}$-order ODEs. The efficiency of the obtained results is validated through selected problems in the literature. It is found that several existing solutions can be deduced as special cases of our generalized one. Moreover, the present results may be invested for similar future problems in fluid mechanics, especially nanofluids.
\end{abstract}

\section{Introduction}

The field of nanofluid is of great importance in industry, engineering, and physics. The distributions of temperature and nanoparticles concentration of such fluids are originally governed by PDEs which can be transformed into ODEs [1-9]. Such ODEs are, basically, subjected to boundary conditions (BCs) given at infinity. In Refs. [10-16], the authors implemented several numerical/analytical methods to solve such types of problems. However, the approaches [10-16] need a massive computational work to obtain accurate solution because of the difficulty of applying the BC at infinity. In addition, it was shown in the literature [17-19] that

Received July $5^{\text {th }}, 2020$; accepted July 27 $7^{\text {th }}, 2020$; published August $20^{\text {th }}, 2020$.

2010 Mathematics Subject Classification. 34B10.

Key words and phrases. ordinary differential equation; hypergeometric series; boundary value problem; exact solution; nanofluid.

(C)2020 Authors retain the copyrights of their papers, and all open access articles are distributed under the terms of the Creative Commons Attribution License. 
some of the approximate methods have drawbacks. Therefore, solving BVPs with singularities is a challenge. Usually, the BCs at infinity are transformed to new finite ones by applying certain substitutions. Accordingly, the coefficients of the ODEs become polynomials. Hence, the temperature/nanoparticles concentration of nanofluids are usually special cases of the following class:

$$
z^{\prime \prime}(t)+\left(\frac{P}{t}+Q\right) z^{\prime}(t)+\left(\frac{l}{t^{2}}+\frac{R}{t}\right) z(t)=\alpha t^{n-1}, \quad n>-1, \alpha \in \mathbb{R}
$$

under the BCs:

$$
z(0)=0, \quad z(\delta)=1, \quad \delta \in \mathbb{R}-\{0\}
$$

where $P, Q, l$, and $R$ are physical parameters of the nanofluids [1-8]. The constant $n$ takes a particular value according to the final form of the temperature equation, while $\delta \neq 0$ depends on the final BC. The main objective of this paper is to introduce a direct analysis to exactly solving Eqs. (1.1-1.2). Then, the present generalized results will be invested to construct several exact solutions for some published nanofluids problems as special cases.

\section{ANALYSIS}

Firstly, we rewrite Eq. (1.1) as

$$
t^{2} z^{\prime \prime}(t)+\left(P t+Q t^{2}\right) z^{\prime}(t)+(l+R t) z(t)=\alpha t^{n+1}
$$

Suppose that

$$
z(t)=t^{\gamma} \psi(t)
$$

Accordingly,

$$
z^{\prime}(t)=t^{\gamma-1}\left(t \psi^{\prime}(t)+\gamma \psi(t)\right)
$$

and

$$
z^{\prime \prime}(t)=t^{\gamma-2}\left(t^{2} \psi^{\prime \prime}(t)+2 \gamma t \psi^{\prime}(t)+\gamma(\gamma-1) \psi(t)\right)
$$

respectively. Substituting Eqs. (2.2-2.4) into Eq. (2.1), we have

$$
t^{2} \psi^{\prime \prime}(t)+\left((2 \gamma+P) t+Q t^{2}\right) \psi^{\prime}(t)+\left(\left(\gamma^{2}-\gamma+\gamma P+l\right)+(\gamma Q+R) t\right) \psi(t)=\alpha t^{n-\gamma+1}
$$

Setting

$$
\gamma^{2}-\gamma+\gamma P+l=0
$$


and solving for $\gamma$, we obtain

$$
\gamma=\frac{1-P \pm \sqrt{(1-P)^{2}-4 l}}{2} .
$$

Accordingly, Eq. (2.5) becomes

$$
t \psi^{\prime \prime}(t)+((2 \gamma+P)+Q t) \psi^{\prime}(t)+(\gamma Q+R) \psi(t)=\alpha t^{n-\gamma} .
$$

Assuming that

$$
P_{1}=2 \gamma+P, \quad R_{1}=\gamma Q+R,
$$

then Eq. (2.8) takes the form:

$$
t \psi^{\prime \prime}(t)+\left(P_{1}+Q t\right) \psi^{\prime}(t)+R_{1} \psi(t)=\alpha t^{n-\gamma} .
$$

\section{ExaCt SOLUtion AND CONVERGENCE ANALYSiS}

3.1. Exact solution. Suppose that $\psi_{c}(t)$ and $\psi_{p}(t)$ are complementary and particular solution of Eq. (2.10), respectively, then

$$
\psi(t)=\psi_{c}(t)+\psi_{p}(t)
$$

Following [20], we have

$$
\psi_{c}(t)=\frac{c t^{\mu_{1}+\mu_{2}-1}}{\Gamma\left(\mu_{1}+\mu_{2}\right)}{ }_{1} F_{1}\left[\mu_{1}, \mu_{1}+\mu_{2},-Q t\right],
$$

where ${ }_{1} F_{1}$ is Kummer's function and $c$ is a constant. $\mu_{1}$ and $\mu_{2}$ are defined as

$$
\mu_{1}=1-P_{1}+\frac{R_{1}}{Q}, \quad \mu_{2}=1-\frac{R_{1}}{Q} .
$$

From (2.9) and (3.3), we have

$$
\mu_{1}=1-\gamma-P+\frac{R}{Q}, \quad \mu_{2}=1-\gamma-\frac{R}{Q} .
$$

$\psi_{p}(t)$ can be obtained as (see [20])

$$
\psi_{p}(t)=\frac{\alpha t^{n-\gamma+1}}{(n-\gamma+1)\left(n-\gamma+P_{1}\right)},
$$

such that

$$
R_{1}=-(n-\gamma+1) Q, \quad(n-\gamma+1)\left(n-\gamma+P_{1}\right) \neq 0 .
$$

From (2.9) and (3.6), we have

$$
R=-(n+1) Q, \quad(n-\gamma+1)(n+\gamma+P) \neq 0,
$$


and hence Eq. (2.8) reduces to

$$
t \psi^{\prime \prime}(t)+((2 \gamma+P)+Q t) \psi^{\prime}(t)-(n-\gamma+1) Q \psi(t)=\alpha t^{n-\gamma} .
$$

Inserting $P_{1}$ given by (2.9) into (3.5), we obtain

$$
\psi_{p}(t)=\frac{\alpha t^{n-\gamma+1}}{(n-\gamma+1)(n+\gamma+P)} .
$$

Therefore, the general solution of Eq. (3.8) is given from (3.1) by

$$
\psi(t)=\frac{c t^{\mu_{1}+\mu_{2}-1}}{\Gamma\left(\mu_{1}+\mu_{2}\right)}{ }_{1} F_{1}\left[\mu_{1}, \mu_{1}+\mu_{2},-Q t\right]+\frac{\alpha t^{n-\gamma+1}}{(n-\gamma+1)(n+\gamma+P)},
$$

where $\mu_{1}$ and $\mu_{2}$ are defined by (3.3). Hence, the general solution of the original equation (2.1) such that $R=-(n+1) Q$ is obtained as

$$
z(t)=\frac{c t^{\gamma+\mu_{1}+\mu_{2}-1}}{\Gamma\left(\mu_{1}+\mu_{2}\right)}{ }_{1} F_{1}\left[\mu_{1}, \mu_{1}+\mu_{2},-Q t\right]+\frac{\alpha t^{n+1}}{(n-\gamma+1)(n+\gamma+P)},
$$

for the ODE:

$$
t^{2} z^{\prime \prime}(t)+\left(P t+Q t^{2}\right) z^{\prime}(t)+(l-(n+1) Q t) z(t)=\alpha t^{n+1}
$$

It is noted from (3.11) that the first $\mathrm{BC} z(0)=0$ is automatically satisfied when

$$
\gamma+\mu_{1}+\mu_{2}>1, \quad n>-1, \quad(n-\gamma+1)(n+\gamma+P) \neq 0 .
$$

Applying the second BC in (1.2) on Eq. (3.11), yields

$$
c=\frac{\delta^{1-\gamma-\mu_{1}-\mu_{2}} \Gamma\left(\mu_{1}+\mu_{2}\right)}{{ }_{1} F_{1}\left[\mu_{1}, \mu_{1}+\mu_{2},-Q \delta\right]}\left(1-\frac{\alpha \delta^{n+1}}{(n-\gamma+1)(n+\gamma+P)}\right) .
$$

In such case, the solution given by Eq. (3.11) becomes

$$
\begin{aligned}
z(t)= & \frac{(t / \delta)^{\gamma+\mu_{1}+\mu_{2}-1}{ }_{1} F_{1}\left[\mu_{1}, \mu_{1}+\mu_{2},-Q t\right]}{{ }_{1} F_{1}\left[\mu_{1}, \mu_{1}+\mu_{2},-Q \delta\right]}\left(1-\frac{\alpha \delta^{n+1}}{(n-\gamma+1)(n+\gamma+P)}\right)+ \\
& \frac{\alpha t^{n+1}}{(n-\gamma+1)(n+\gamma+P)} .
\end{aligned}
$$

Therefore

$$
\begin{aligned}
z(t) & =\frac{(t / \delta)^{1-\gamma-P}{ }_{1} F_{1}[-n-\gamma-P, 2-2 \gamma-P,-Q t]}{{ }_{1} F_{1}[-n-\gamma-P, 2-2 \gamma-P,-Q \delta]} \times \\
& \left(1-\frac{\alpha \delta^{n+1}}{(n-\gamma+1)(n+\gamma+P)}\right)+\frac{\alpha t^{n+1}}{(n-\gamma+1)(n+\gamma+P)},
\end{aligned}
$$

provided that

$$
1-\gamma-P>0, \quad n>-1, \quad(n-\gamma+1)(n+\gamma+P) \neq 0 .
$$

It can be verified by direct substitution that the solution (3.16) satisfies Eq. (3.12) and the BCs (1.2). In a sequent section, it is declared that Eq. (3.16) agrees with several existing results at prescribed values of the coefficients $P, Q, l, R$, and the parameter $\delta$. 
3.2. Convergence analysis. In order to prove the convergence of the solution given by Eqs. (3.16-3.17), we begin with the definition of Kummer's function ${ }_{1} F_{1}(a, b, t)$ :

$$
{ }_{1} F_{1}(a, b, t)=\sum_{i=0}^{\infty} \frac{(a)_{i}}{(b)_{i}} \frac{t^{i}}{i !},
$$

where $(a)_{i}$ is Pochhammer symbol defined as

$$
(a)_{i}=a(a+1)(a+2) \ldots(a+i-1), i=1,2,3, \ldots, \quad(a)_{0}=1 .
$$

The series (3.18) is not defined for $b=0,-1,-2,-3, \ldots$, and if $a$ is a negative integer, the series truncates.

Theorem 1: Let $b$ is neither a negative integer nor zero, then ${ }_{1} F_{1}(a, b,-Q t)$ converges for all (finite) $t$ and finite $Q$.

Proofs: From the definition $(3.18),{ }_{1} F_{1}(a, b,-Q t)$ is given as

$$
{ }_{1} F_{1}(a, b,-Q t)=\sum_{i=0}^{\infty} \frac{(a)_{i}}{(b)_{i}} \frac{(-Q t)^{i}}{i !} .
$$

Since $b$ is neither a negative integer nor zero, then the series (3.20) is defined and its general term $v_{i}(x)$ is given by

$$
v_{i}(x)=\frac{(a)_{i}}{(b)_{i}} \frac{(-Q t)^{i}}{i !}
$$

Implementing the ratio test, we have

$$
\begin{aligned}
\lim _{i \rightarrow \infty}\left|\frac{v_{i+1}(x)}{v_{i}(x)}\right| & =\lim _{i \rightarrow \infty}\left|\frac{(a)_{i+1}}{(b)_{i+1}} \frac{(-Q t)^{i+1}}{(i+1) !} \times \frac{(b)_{i}}{(a)_{i}} \frac{i !}{(-Q t)^{i}}\right|, \\
& =\lim _{i \rightarrow \infty}\left|\frac{(a)_{i}(a+i)}{(b)_{i}(b+i)} \frac{(-Q)^{i+1}(t)^{i+1}}{(i+1)(i !)} \times \frac{(b)_{i}}{(a)_{i}} \frac{i !}{(-Q)^{i}(t)^{i}}\right|, \\
& =\lim _{i \rightarrow \infty}\left|\frac{(a+i)}{(b+i)(i+1)}\right| \times|-Q||t| .
\end{aligned}
$$

For finite $t$ and finite $Q$, we have from (3.22) that

$$
\lim _{i \rightarrow \infty}\left|\frac{v_{i+1}(x)}{v_{i}(x)}\right|=0
$$

and hence, ${ }_{1} F_{1}(a, b,-Q t)$ is convergent.

Lemma 1: For finite $\delta$ and $Q$, the solution given by Eqs. (3.16-3.17) converges if $(2-2 \gamma-P)$ is neither a negative integer nor zero.

Proofs: Let $a=-n-\gamma-P$ and $b=2-2 \gamma-P$, then the solution in Eqs. (3.16-3.17) takes the form:

$$
z(t)=\frac{(t / \delta)^{1-\gamma-P}{ }_{1} F_{1}[a, b,-Q t]}{{ }_{1} F_{1}[a, b,-Q \delta]}\left(1+\frac{\alpha \delta^{n+1}}{(n-\gamma+1) a}\right)-\frac{\alpha t^{n+1}}{(n-\gamma+1) a},
$$

such that

$$
1-\gamma-P>0, \quad n>-1, \quad(n-\gamma+1) a \neq 0 .
$$


Since $b=2-2 \gamma-P$ is neither a negative integer nor zero, then $z(t)$ in (36) is defined. Also, since $Q$ is finite and $t$ is finite in the domain of the problem, $t \in[0, \delta]$, then ${ }_{1} F_{1}(a, b,-Q t)$ is convergent by Theorem 1. Also, ${ }_{1} F_{1}(a, b,-Q \delta)$ is convergent because $\delta$ is finite and therefore the solution given by Eqs. (3.16-3.17) or its equivalent form (3.24-3.25) converges.

\section{Applichtions}

4.1. At $l=0, \alpha \neq 0, n>-1$. At $l=0, \gamma$ in (2.7) reduces to

$$
\gamma=\frac{1-P \pm|1-P|}{2}
$$

For $P<1$, we have $\gamma=1-P$ or $\gamma=0$. However, $\gamma=1-P$ doesn't satisfy the first condition in (3.25) which is $1-\gamma-P>0$. Also, for $P>1$, we have $\gamma=0$ or $\gamma=1-P$. Hence, $\gamma=0$ is the only acceptable value when the special case $l=0$ is considered. At such case $(l=0)$, Eq. (3.12) reduces to

$$
t z^{\prime \prime}(t)+(P+Q t) z^{\prime}(t)-((n+1) Q) z(t)=\alpha t^{n}
$$

and its solution comes by setting $\gamma=0$ in (3.16) which is

$$
\begin{aligned}
z(t) & =\frac{(t / \delta)^{1-P}{ }_{1} F_{1}[-n-P, 2-P,-Q t]}{{ }_{1} F_{1}[-n-P, 2-P,-Q \delta]}\left(1-\frac{\alpha \delta^{n+1}}{(n+1)(n+P)}\right)+ \\
& \frac{\alpha t^{n+1}}{(n+1)(n+P)} .
\end{aligned}
$$

The result obtained by Eq. (4.3) agrees with the published solution in literature (Ref. [20], equation 31). Consequently, the solution in literature is a special case of our analysis when $l=0$. In addition, the solution (40) converges if $(2-P)$ is neither a negative integer nor zero, i.e., $P \neq 2,3,4, \ldots$, or $P \neq(j+1) \forall j \in \mathbb{Z}^{+}$.

4.2. At $l=0, \alpha \neq 0, n=1, \delta=1$. The temperature equation for the Marangoni boundary layer was obtained by Khaled [21] as

$$
t z^{\prime \prime}(t)+\left(l_{1}-m t\right) z^{\prime}(t)+2 m z(t)=-\lambda t
$$

subject to

$$
z(0)=0, \quad z(1)=1 .
$$

At $n=1$, Eq. (4.2), in the previous section, becomes

$$
t z^{\prime \prime}(t)+(P+Q t) z^{\prime}(t)-2 Q z(t)=\alpha t .
$$

Comparing (4.4) with (4.2) and (4.5) with (1.2), we find that

$$
P=l_{1}, \quad Q=-m, \quad \alpha=-\lambda, \quad \delta=1 .
$$


Substituting these values of parameters into (4.3), we have

$$
y(t)=\left(1+\frac{\lambda}{2\left(l_{1}+1\right)}\right) \frac{t^{1-l_{1}}{ }_{1} F_{1}\left[-1-l_{1}, 2-l_{1}, m t\right]}{{ }_{1} F_{1}\left[-1-l_{1}, 2-l_{1}, m\right]}-\frac{\lambda t^{2}}{2\left(l_{1}+1\right)},
$$

which is the same obtained result in Ref. [21]. As shown in the previous subsection, the solution (4.8) converges if $l_{1} \neq(j+1) \forall j \in \mathbb{Z}^{+}$.

4.3. At $l=0, \alpha=0, \delta=\frac{\mathbf{S c}}{\beta^{2}}$. Qasim [22] obtained the mass transfer equation:

$$
t z^{\prime \prime}(t)+\left(1-\frac{\mathrm{Sc}}{\beta^{2}}+t\right) z^{\prime}(t)-m z(t)=0,
$$

for a Jeffrey fluid with heat source/sink, where

$$
z(0)=0, \quad z\left(\frac{\mathrm{Sc}}{\beta^{2}}\right)=1 .
$$

$\mathrm{Sc}>0$ is the Schmidt parameter and $\beta$ is a positive parameter. It then follows that

$$
P=1-\frac{\mathrm{Sc}}{\beta^{2}}, \quad Q=1, n=m-1, \quad \alpha=0, \quad \delta=\frac{\mathrm{Sc}}{\beta^{2}} .
$$

Inserting these values into (4.3), we obtain

$$
z(t)=\left(\frac{\beta^{2}}{\mathrm{Sc}} t\right)^{\frac{\mathrm{Sc}}{\beta^{2}}} \frac{{ }_{1} F_{1}\left[\frac{\mathrm{Sc}}{\beta^{2}}-m, \frac{\mathrm{Sc}}{\beta^{2}}+1,-t\right]}{{ }_{1} F_{1}\left[\frac{\mathrm{Sc}}{\beta^{2}}-m, \frac{\mathrm{Sc}}{\beta^{2}}+1,-\frac{\mathrm{Sc}}{\beta^{2}}\right]} .
$$

The solution (4.12) can be verified by substitution. It should be mentioned that the solution obtained by Qasim $[22]$ as

$$
z(t)=\left(\frac{\beta^{2}}{\mathrm{Sc}} t\right)^{\frac{\mathrm{Sc}}{\beta^{2}}} \frac{{ }_{1} F_{1}\left[\frac{\mathrm{Sc}}{\beta^{2}}-m, 2 \frac{\mathrm{Sc}}{\beta^{2}}+1,-t\right]}{{ }_{1} F_{1}\left[\frac{\mathrm{Sc}}{\beta^{2}}-m, 2 \frac{\mathrm{Sc}}{\beta^{2}}+1,-\frac{\mathrm{Sc}}{\beta^{2}}\right]},
$$

does not satisfy Eq. (4.9). Since $\beta>0$ and $\mathrm{Sc}>0$, then the magnitude $\left(\frac{\mathrm{Sc}}{\beta^{2}}+1\right)$ is never a zero or a negative integer. Hence, the solution (4.12) converges for all positive values of $\beta$ and Sc.

4.4. At $l \neq 0, \alpha=0, \delta=\frac{\mathbf{P r}}{\beta^{2}}$. The following heat transfer equation

$$
t^{2} z^{\prime \prime}(t)+\left(\left(1-\frac{\operatorname{Pr}}{\beta^{2}}\right) t+t^{2}\right) z^{\prime}(t)-\left(m t-\frac{\gamma_{1}}{\beta^{2}}\right) z(t)=0,
$$

was also obtained by Qasim [22], where

$$
z(0)=0, \quad z\left(\frac{\operatorname{Pr}}{\beta^{2}}\right)=1
$$

$\operatorname{Pr}>0$ is the Prandtl number, $\beta>0$, and $\gamma_{1}$ is the heat generation/absorption parameter. In this case, we have

$$
P=1-\frac{\operatorname{Pr}}{\beta^{2}}, \quad Q=1, n=m-1, \quad \alpha=0, \quad l=\frac{\gamma_{1}}{\beta^{2}}, \quad \delta=\frac{\operatorname{Pr}}{\beta^{2}}
$$


At $l=\frac{\gamma_{1}}{\beta^{2}}, \gamma$ is given by

$$
\gamma=\frac{1}{2}\left(\frac{\operatorname{Pr}}{\beta^{2}}-\sqrt{\frac{\operatorname{Pr}^{2}}{\beta^{4}}-\frac{4 \gamma_{1}}{\beta^{2}}}\right),
$$

which be written as

$$
\gamma=\frac{1}{2}\left(\frac{\operatorname{Pr}}{\beta^{2}}-\sqrt{\frac{\operatorname{Pr}^{2}}{\beta^{4}}-\frac{4 \gamma_{1}}{\beta^{2}}}\right)=k_{1}-k_{2},
$$

where

$$
k_{1}=\frac{\operatorname{Pr}}{2 \beta^{2}}, \quad k_{2}=\frac{\sqrt{\operatorname{Pr}^{2}-4 \gamma_{1} \beta^{2}}}{2 \beta^{2}} .
$$

Hence, the solution of the present model is

$$
z(t)=\left(\frac{\beta^{2}}{\operatorname{Pr}} t\right)^{k_{1}+k_{2}} \frac{{ }_{1} F_{1}\left[k_{1}+k_{2}-m, 2 k_{1}+1,-t\right]}{{ }_{1} F_{1}\left[k_{1}+k_{2}-m, 2 k_{1}+1,-\frac{\operatorname{Pr}}{\beta^{2}}\right]},
$$

which is the same exact solution obtained by Qasim [22]. Since $\beta$ and $\operatorname{Pr}$ are always positives, then $\left(2 k_{1}+1\right)=$ $\left(\frac{\mathrm{Pr}}{\beta^{2}}+1\right)$ is never a zero or a negative integer. Hence, the solution (4.20) converges for all positive values of $\beta$ and Pr.

4.5. At $l \neq 0, \alpha=0, \delta=-\mathbf{S c}^{*}$. Kameswaran et. al [2] obtained following equation for the mass transfer of nanofluids:

$$
t^{2} z^{\prime \prime}(t)+\left(\left(1-\mathrm{Sc}^{*}\right) t-t^{2}\right) z^{\prime}(t)+\left(2 t-\gamma_{2} \mathrm{Sc}^{*}\right) z(t)=0
$$

subject to

$$
z(0)=0, \quad z\left(-\mathrm{Sc}^{*}\right)=1,
$$

where $\gamma_{2}$ is the parameter of scaled chemical reaction and $\mathrm{Sc}^{*}$ is the modified Schmidt number. Thus

$$
P=1-\mathrm{Sc}^{*}, \quad Q=-1, n=1, \quad \alpha=0, \quad l=-\gamma_{2} \mathrm{Sc}^{*}, \quad \delta=-\mathrm{Sc}^{*} .
$$

At $l=-\gamma_{2} \mathrm{Sc}^{*}$, we obtain $\gamma$ as

$$
\gamma=\frac{1}{2}\left(\mathrm{Sc}^{*}-\sqrt{\left(\mathrm{Sc}^{*}\right)^{2}+4 \gamma_{2} \mathrm{Sc}^{*}}\right)
$$

or

$$
\gamma=\frac{c_{1}-d_{1}}{2}, \quad c_{1}=\mathrm{Sc}^{*}, \quad d_{1}=\left(\mathrm{Sc}^{*}\right)^{2}+4 \gamma_{2} \mathrm{Sc}^{*} .
$$

The solution of the present model is in the form:

$$
z(t)=\left(-\frac{t}{\mathrm{Sc}^{*}}\right)^{\frac{c_{1}+d_{1}}{2}} \frac{{ }_{1} F_{1}\left[\frac{c_{1}+d_{1}}{2}-2, d_{1}+1, t\right]}{{ }_{1} F_{1}\left[\frac{c_{1}+d_{1}}{2}-2, d_{1}+1,-\mathrm{Sc}^{*}\right]},
$$


which agrees with Kameswaran et. al [2]. According to the physical values taken by the authors [2], the magnitude $d_{1}+1=\left(\mathrm{Sc}^{*}\right)^{2}+4 \gamma_{2} \mathrm{Sc}^{*}+1$ is always positive and this admits the convergence of the solution (4.26).

\section{Conclusion}

In this paper, a general solution was obtained for a class of singular BVPs arise in the field of nanofluids. The solution was derived in terms of the hypergeometric series. The studied class reduced to several published physical models at particular choices of the involved parameters. The obtained solutions were compared with the corresponding results of several models in the literature. It was found that the results in the literature were recovered as special cases of the current ones. Furthermore, this work can be extended in the near future to deal with the recently published physical models [23-25].

Conflicts of Interest: The author(s) declare that there are no conflicts of interest regarding the publication of this paper.

\section{REFERENCES}

[1] M.A.A. Hamad, Analytical solution of natural convection flow of a nanofluid over a linearly stretching sheet in the presence of magnetic field, Int. Commun. Heat Mass Transfer 38 (2011), 487-492.

[2] P.K. Kameswaran, M. Narayana, P. Sibanda and P.V.S.N. Murthy, Hydromagnetic nanofluid flow due to a stretching or shrinking sheet with viscous dissipation and chemical reaction effects, Int. J. Heat Mass Transfer, 55 (2012), 7587-7595.

[3] E.H. Aly and A. Ebaid, Exact analytical solution for suction and injection flow with thermal enhancement of five nanofluids over an isothermal stretching sheet with effect of the slip model: a comparative study, Abstr. Appl. Anal. 2013 (2013), Article ID 721578.

[4] E.H. Aly and A. Ebaid, New exact solutions for boundary-layer flow of a nanofluid past a stretching sheet, J. Comput. Theor. Nanosci. 10 (11) (2013), 2591-2594.

[5] W.A. Khan, Z.H. Khan and M. Rahi, Fluid flow and heat transfer of carbon nanotubes along a flat plate with Navier slip boundary. Appl. Nanosci. 4 (2014), 633-641.

[6] A. Ebaid, F. Al Mutairi and S.M. Khaled, Effect of velocity slip boundary condition on the flow and heat transfer of $\mathrm{Cu}$-water and TiO2-water nanofluids in the presence of a magnetic field, Adv. Math. Phys. 2014 (2014), Article ID 538950.

[7] A. Ebaid and M. Al Sharif, Application of Laplace transform for the exact effect of a magnetic field on heat transfer of carbon-nanotubes suspended nanofluids, Z. Naturforsch. A. 70 (6) (2015), 471-475.

[8] E.R. EL-Zahar, A.M. Rashad and A.M. Gelany, Studying High Suction Effect on Boundary-Layer Flow of a Nanofluid on Permeable Surface via Singular Perturbation Technique, J. Comput. Theor. Nanosci. 12 (11) (2015), 4828-4836.

[9] E.H. Aly and A. Ebaid, Exact analysis for the effect of heat transfer on MHD and radiation Marangoni boundary layer nanofluid flow past a surface embedded in a porous medium, J. Molecular Liquids, 215 (2016), 625-639.

[10] J.P. Boyd, Pade-approximant algorithm for solving nonlinear ordinary differential equation boundary value problems on an unbounded domain, Comput. Phys. 11 (3) (1997), 299-303. 
[11] A.M. Wazwaz, The modified decomposition method and Pade approximants for solving the Thomas-Fermi equation, Appl. Math. Comput. 105 (1) (1999), 11-19.

[12] A.M. Wazwaz, The modified decomposition method and Pade approximants for a boundary layer equation in unbounded domain, Appl. Math. Comput. 177 (2) (2006), 737-744.

[13] E.R. EL-Zahar and Y.S. Hamed, An Algorithm for Solving Second Order Nonlinear Singular Perturbation Boundary Value Problems, J. Mod. Methods Numer. Math. 2 (1-2) (2011), 21-31.

[14] E.R. EL-Zahar, Approximate Analytical Solutions for Singularly Perturbed Boundary Value Problems by Multi-Step Differential Transform Method, J. Appl. Sci. 12 (19) (2012), 2026-2034.

[15] E.R. El-Zahar, Applications of Adaptive Multi step Differential Transform Method to Singular Perturbation Problems Arising in Science and Engineering, Appl. Math. Inform. Sci. 9 (1) (2015), 223-232.

[16] E.R. EL-Zahar and S.M.M. EL-Kabeir, Approximate Analytical Solution of Nonlinear Third-Order Singularly Perturbed BVPs using Homotopy Analysis-Pade Method, J. Comput. Theor. Nanosci. 13 (2016), 8917-8927.

[17] A. Ebaid and E.H. Aly, Exact analytical solution of the peristaltic nanofluids flow in an asymmetric channel with flexible walls: Application to cancer treatment, Comput. Math. Methods Med. 2013 (2013), Article ID 825376.

[18] A. Ebaid, Remarks on the homotopy perturbation method for the peristaltic flow of Jeffrey fluid with nano-particles in an asymmetric channel, Comput. Math. Appl. 68 (3) (2014), 77-85.

[19] A. Ebaid and S.M. Khaled, An Exact Solution for a Boundary Value Problem with Application in Fluid Mechanics and Comparison with the Regular Perturbation Solution, Abstr. Appl. Anal. 2014 (2014), Article ID 172590.

[20] A. Ebaid, A.M. Wazwaz, E. Alali and B. Masaedeh, Hypergeometric Series Solution to a Class of Second-Order Boundary Value Problems via Laplace Transform with Applications to Nanouids, Commun. Theor. Phys. 67 (2017), 231.

[21] S.M. Khaled, The exact effects of radiation and joule heating on magnetohydrodynamic Marangoni convection over a flat surface, Therm. Sci. 22 (2018), 63-72.

[22] M. Qasim, Heat and mass transfer in a Jeffrey fluid over a stretching sheet with heat source/sink, Alexandria Eng. J. 52 (2013), 571-575.

[23] A. Ebaid, E. Alali, H.S. Ali, The exact solution of a class of boundary value problems with polynomial coefficients and its applications on nanofluids, J. Assoc. Arab Univ. Basic Appl. Sci. 24 (2017), 156-159.

[24] H.S. Ali, Elham Alali, A. Ebaid and F.M. Alharbi, Analytic Solution of a Class of Singular Second-Order Boundary Value Problems with Applications, Mathematics, 7 (2019), 172.

[25] Q.M. Al-Mdallal, N. Indumathi, B. Ganga and A.K. Abdul Hakeem, Marangoni radiative effects of hybrid-nanofluids flow past a permeable surface with inclined magnetic field, Case Stud. Therm. Eng. 17 (2020), 100571. 